\title{
The Delaware Asteroseismic Research Center: Convection in Pulsating White Dwarfs
}

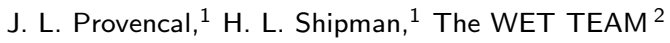 \\ ${ }^{1} \mathrm{Mt}$. Cuba Observatory and the University of Delaware, \\ Dept. of Physics and Astronomy, Newark, DE 19716, USA \\ 2 ww. physics.udel.edu/darc/wet/
}

\begin{abstract}
We introduce the Delaware Asteroseismic Research Center (DARC), a new initiative sponsored by Mt. Cuba Observatory and supported by the Department of Physics and Astronomy at the University of Delaware. DARC's mission is to promote and facilitate international collaboration in the field of stellar seismology. We present preliminary results from XCOV25, the first observing run sponsored by DARC. XCOV25's primary target was GD358, the prototype DB pulsating white dwarf. The scientific goals focus on expanding our understanding of stellar convection.
\end{abstract}

\section{Introducing DARC}

The light from stellar sources, be it detected using a $0.6 \mathrm{~m}$ or a $10 \mathrm{~m}$ telescope, originates from their surfaces. Stellar interiors cannot be directly observed. Asteroseismology offers the best method to indirectly peer below stellar surfaces, using pulsations to determine internal structure. Multisite photometric and spectroscopic campaigns are the primary observational tool, providing the uninterrupted observations and lengthy timebase necessary to resolve complicated pulsation spectra of many variable stars.

The Whole Earth Telescope (WET), founded in the 1980s by R. E. Nather and D. E. Winget (Nather et al. 1990), took multisite campaigns to the next level. WET's purpose is to obtain continuous coverage of a primary target, and to maximize the use of telescope time to cover additional targets by providing real-time data reduction and an interactive headquarters. In 2004, WET's governing council gave permission to one of us (HLS) to explore the possibility of private funding to support WET. The result was the formation of the Delaware Asteroseismic Research Center (DARC) in 2005. WET moved from lowa to Delaware and completed the first WET run supported by DARC (XCOV25) in May of 2006. Preliminary results from this run are reported below.

DARC is sponsored by Mt. Cuba Observatory in collaboration with the Department of Physics and Astronomy at the University of Delaware. Our purpose is to support and promote international collaboration in the field of stellar seismology. The DARC director is supported by an Advisory board. We encourage development of instrumentation and software, observing techniques, and science goals. To this end, we are in the process of expanding our theoretical and technical support. Targets for WET runs or campaign support should not be limited to white dwarfs and can be submitted at any time through the DARC website (www.physics.udel.edu/darc/proposal.html). Submit approximately one page describing the proposed target and scientific justification. If the target and science goals require a full WET run with headquarters, please justify your reasoning. WET runs require about a year of organization, so submit targets early. The Director and the Advisory Board will debate/discuss the proposal, and respond with any additional questions. We are also in the progress of creating an on-line data archive. Many older PMT runs are already 
available at www.physics.udel.edu/darc/archives.html. We are extremely interested in feedback from the community. If you have suggestions, please send them along via the feedback form on the website, or mail DARC at darcdelaware@gmail.com. A detailed discussion of available opportunities can be found in Provencal et al. (2007) or at our website at www.physics.udel.edu/darc.

\section{Looking at Convection in Pulsating White Dwarfs}

Convection is an important means of energy transfer for virtually all stars, yet convection remains one of the largest uncertainties in stellar modelling. Montgomery (2005) presents a method by which precise observations of light curves of certain types of variable stars can be used to determine parameters characterizing the convection zone of a particular star.

In general, stellar pulsations are described in terms of spherical harmonics, and are assigned three indices $(\mathrm{k}, \ell$, and $\mathrm{m})$ that describe the pulsation. The quantities $\ell$ and $\mathrm{m}$ describe the angular geometry of a given nonradial pulsation. The radial component is defined by $\mathrm{k}$.

Montgomery (2005) and Montgomery (2007) outline the theoretical details of this technique to investigate white dwarf convection zones. Observationally, it requires 1 ) a nonlinear pulsator 2) knowledge of the $\mathrm{k}, \ell$ and $\mathrm{m}$ values of the pulsations, and 3) a very high signalto-noise light curve (4-5 hours minimum for white dwarfs).

We chose GD358 as a good target for this technique. It is a well studied, large amplitude nonlinear pulsator with known $\mathrm{k}, \ell$ and $\mathrm{m}$ values (Winget et al. 1994). We organized a WET campaign in May 2006 with two goals: 1) to acquire a high signal to noise light curve, 2) to obtain contemporary frequency, phase, and amplitude information.

\section{The Observations}

Twenty-two telescopes participated in the run, from May 12 to June 14 (a complete list of participants can be found at www.physics.udel.edu/darc). We obtained over 282 hours of observations, achieving $73 \%$ coverage during the main portion of the run. The observations were acquired with a mixture of CCD and PMT photometers and were optimized to use identical comparison stars where possible. The majority of sites used a BG40 filter to normalize spectral response. The data were reduced using the techniques described in Nather et al. (1990) and Kepler et al. (2003). Figure 1 presents a portion of the complete light curve.

Figure 2 presents the Fourier Transform (FT) of the entire data set. Multi-frequency analysis was carried out using the Period04 software package described by Lenz \& Breger (2005). We find power at the $\ell=1$ modes of $k=21,19,18,15,14,12,9$ and 8 , albeit with different amplitudes than in previous years. In addition, we detect $\approx 100$ combination frequencies, a few of which are labelled in Fig. 2.

\section{The Fourier Transform}

Table 1 lists a preliminary sampling of frequency identifications. The dominant mode is $k=18(1234.124 \mu \mathrm{Hz}, 810.291 \mathrm{~s})$ with an average amplitude of $24.04 \mathrm{mma}$. The mode $k=18$ was detected in previous observations but not as the dominant frequency. Kepler et al. (2003) detected significant power at $1255.4 \mu \mathrm{Hz}$, but speculated that this represents an $\ell=2$ mode. We do not detect power at $1255 \mu \mathrm{Hz}$.

The mode $k=12$ has the second largest amplitude at over $16 \mathrm{mma}$. This mode was detected in 1990, 1994, 1996, and 2000 but never with amplitudes significantly above $1 \mathrm{mma}$. Both $k=18$ and $k=12$ exhibit complex multiplet structure which is undergoing further analysis.

The modes $k=9$ and $k=8$ are both present with frequencies and amplitudes similar to previous measurements. The multiplet splitting is $3.8 \mu \mathrm{Hz}$. 


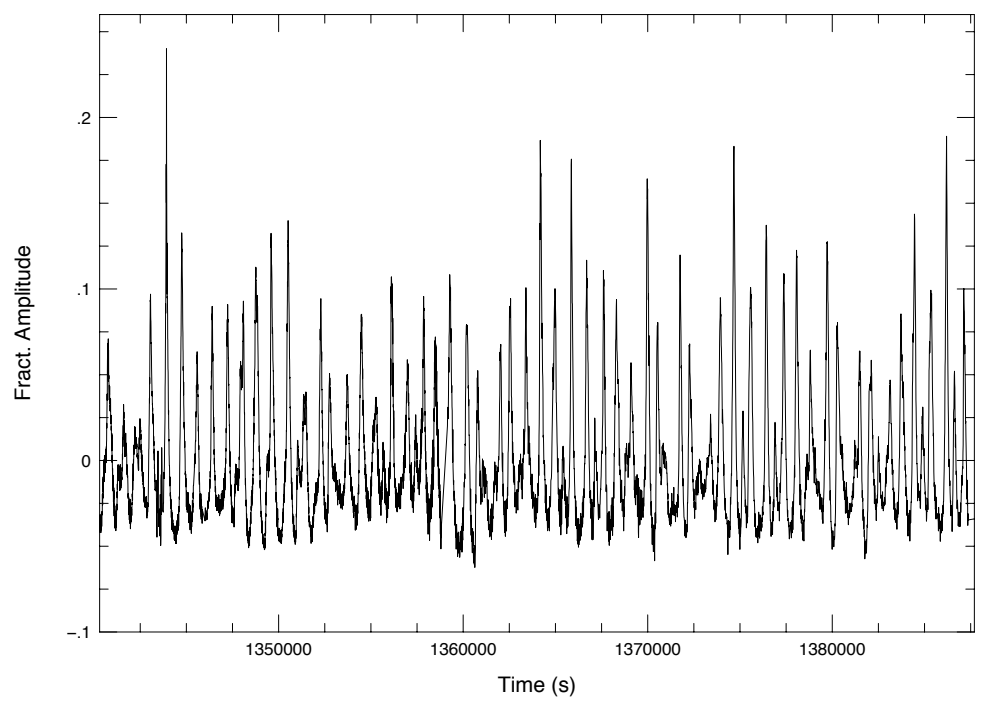

Figure 1: Portion of the XCOV25 light curve of GD 358

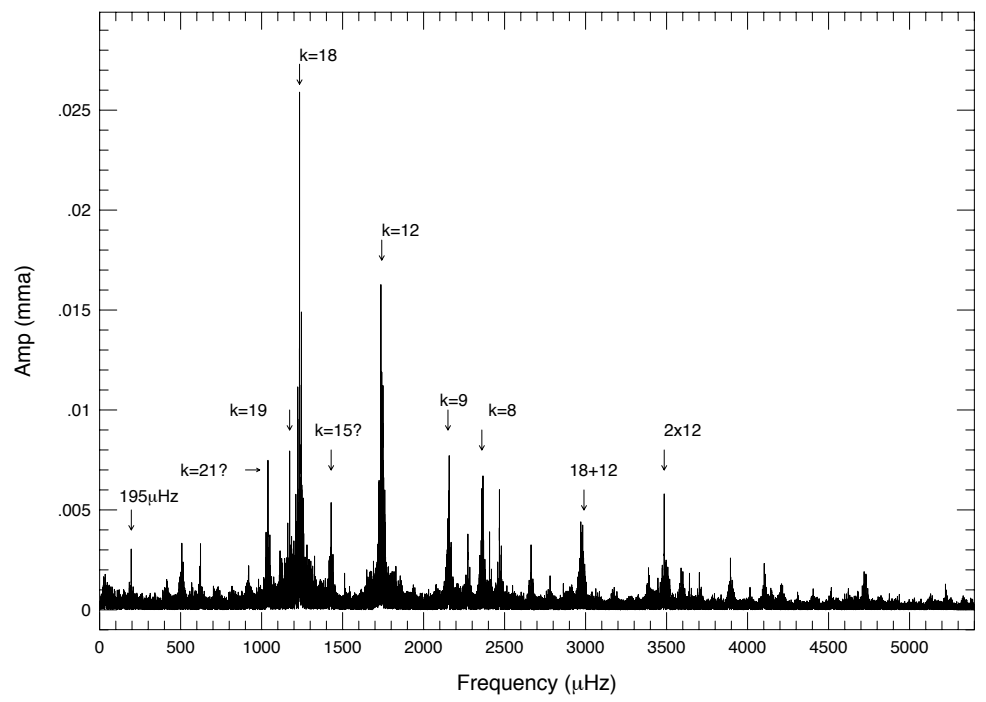

Figure 2: Fourier Transform of GD358 (XCOV25) 
Table 1: Preliminary frequency solution for GD 358 from XCOV25. The third column gives $\mathrm{k}$ values, identifies harmonics, or identifies sum/difference frequencies. The 1223 and $1245-\mu \mathrm{Hz}$ "components" of the $k=18$ mode may be combination frequencies or be due to amplitude modulation.

\begin{tabular}{ccc}
\hline $\begin{array}{c}\text { Frequency }(\mu \mathrm{Hz}) \\
\pm 0.001\end{array}$ & $\begin{array}{c}\text { Amplitude (mma) } \\
\pm 0.07\end{array}$ & Note \\
\hline 195.0685 & 2.73 & $(k=18)-(k=21) ?$ \\
617.4310 & 2.04 & $(k=18) / 2$ \\
1039.0758 & 7.94 & $k=21 ?$ \\
& & $k=18)-195 \mu \mathrm{Hz}$ \\
1173.0152 & 7.24 & $k=19$ \\
1222.9457 & 4.30 & $k=18$ \\
1228.7918 & 5.06 & $k=18$ \\
1234.1243 & 24.03 & $k=18=0$ \\
1239.5107 & 4.93 & $k=18$ \\
1245.2199 & 4.90 & $k=15 ?$ \\
1429.2096 & 5.63 & $k=18)+195 \mu \mathrm{Hz}$ \\
& & $k=14$ \\
1512.1414 & 1.80 & $k=12$ \\
1736.3016 & 16.35 & $k=12$ \\
1741.6663 & 11.01 & $k=12$ \\
1746.9094 & 1.85 & $k=12$ \\
1749.0833 & 11.84 & $k=9$ \\
2150.3934 & 4.09 & $k=9, m=0$ \\
2154.2235 & 5.51 & $k=9$ \\
2158.0740 & 7.18 & $k=8$ \\
2273.6910 & 4.23 & $2 \times(k=18)-195 \mu \mathrm{Hz}$ \\
2359.0525 & 5.95 & $k=8$ \\
2363.0582 & 1.64 & $2 \times(k=18), m=0$ \\
2366.5243 & 6.60 & $(k=18)+195 \mu \mathrm{Hz}$ \\
2468.2817 & 5.19 & $(k=18)+(k=12)$ \\
2663.3676 & 2.95 & \\
2975.8137 & 3.47 &
\end{tabular}

We find numerous combination frequencies, in particular a complex area near $3000 \mu \mathrm{Hz}$. The largest peak is $2975 \mu \mathrm{Hz}$, corresponding to a combination of the dominant frequencies of $k=18$ and $k=12$. Additional peaks in this region correspond to combinations of other multiplets of these two modes.

The Role of Amplitude Modulation

Comparison of our results with those from previous years naturally results in the conclusion that amplitude modulation plays a role in GD358. Montgomery's nonlinear fitting technique requires knowledge of the frequencies present in the light curve. We are interested in identifying actual modes and excluding artifacts due to amplitude modulation.

Drawing an analogy with radio, the general idea supposes a constant carrier wave modulated by an amplitude modulation frequency which may or not be variable itself. In the simplest case, the FT of such a signal will contain the carrier frequency, two sidebands ( \pm the modulation frequency) and the modulation frequency itself. Armed with this simplistic model, we looked for this signature in the FT. If we assume that the carrier frequency is $k=18$, then we find two peaks, at 1429.210 and 1039.076 that are separated from $k=18$ by $195 \mu \mathrm{Hz}$. Interestingly, we also find a significant peak at $195.685 \mu \mathrm{Hz}$. We tentatively identified the power at $1429.210 \mu \mathrm{Hz}$ as $k=15$, but this frequency is shifted by $\approx 2 \mu \mathrm{Hz}$ from previous 
measurements. Amplitude modulation would naturally explain this shift. In addition, we are exploring similar signatures surrounding $k=18$ 's first harmonic and combination frequencies near $3000 \mu \mathrm{Hz}(k=18+k=12)$.

We have also looked at the FTs of subdivisions of the light curve to establish timescales of modulation. The FT is stable over timescales of about one week, but starts to exhibit amplitude variation on shorter timescales (a few days). The mode $k=18$ varies in amplitude from 28 to $20 \mathrm{mma}$, while $k=12$ varies from 10 to $28 \mathrm{mma}$. The beat time between $k=12$ and $k=18$ is of order half an hour, so this cannot account for the variations we observe. Work is ongoing on the implications.

\section{Light Curve Fitting and Convection}

Montgomery (2007) outlines the technique of light curve fitting. Our preliminary fits use 15 independent modes. The best linear fit solution, including just the 15 frequencies and excluding combination frequencies, has residuals of $\sigma^{2}=3.4 \times 10^{-4}$. The best linear fit including combination frequencies, introducing 112 additional parameters, has residuals of $\sigma^{2}=1.3 \times 10^{-4}$. Figure 3 shows the best nonlinear fit, which includes the 15 independent frequencies and 3 additional convection parameters. The residuals for this fit are $\sigma^{2}=$ $1.3 \times 10^{-4}$.

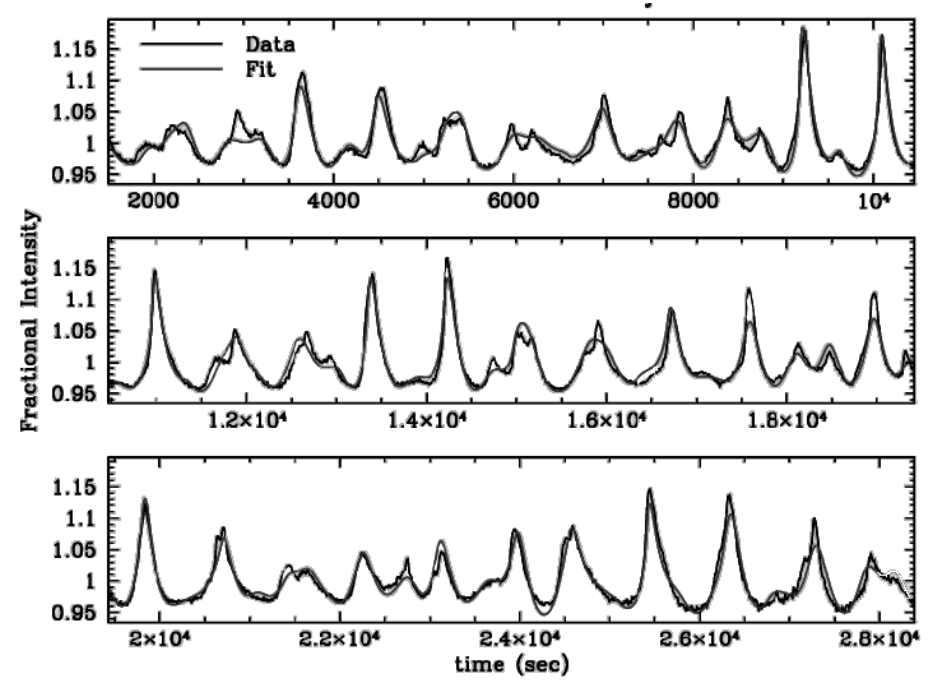

Figure 3: Best fit, nonlinear solution (3 additional parameters)

\section{Conclusions}

This data set has given us new insight into GD358, stellar convection and pulsation/convection interaction, but raised new questions as well. The role of the convection zone in nonlinear pulsators seems clear. For example, convection does not play a role in the DOV pulsators, and no combination frequencies are detected in these stars. Going back to basic physics demonstrations, water in a tank will reflect off the tank walls. In a star, the bottom of the convection zone plays the role of the wall. Yet, because the star is pulsating, the convection 
zone is constantly changing. For an $\mathrm{m}=0$ mode, the poles appear to recede, but the equator does not. In other words, the convection zone does not always form a spherical reflective surface. Could this explain the difference in behaviour of the various modes in GD358? Can this explain the apparent changes in mode trapping we observe? What is the role of amplitude modulation? What physical process could modulate one mode and not others?

The nonlinear light curve fitting technique allows us to probe the convection zone of stars other than our sun. We now have two DBVs spanning the helium instability strip and one DAV probing the DA instability strip. Our future work includes searching for additional targets to map both instability strips completely, and expanding this technique to apply to other types of variable stars.

Acknowledgments. DARC acknowledges the support of the Crystal Trust Foundation and Mt. Cuba Observatory. We would also like to thank everyone involved in the network for their time and support.

\section{References}

Kepler S. O., Nather R. E., Winget D. E., et al., 2003, A\&A, 401, 639

Lenz P., Breger M., 2005, Comm. Asteroseis., 146, 53

Nather R. E., Winget D. E., Clemens J. C., Hansen C. J., Hine B. P., 1990, ApJ, 361, 309

Montgomery M. H., 2005, ApJ, 633, 1142

Montgomery M. H., 2007, Comm. Asteroseis., 150, 253

Provencal J. L., Shipman H. L., Montgomery M. H., et al., 2007, in Napiwotzki R., Burleigh M., eds, ASP Conf. Ser., 15th European Workshop on White Dwarfs. Astron. Soc. Pac., San Francisco, in press

Winget D. E., Nather R. E., Clemens J. C., et al., 1994, ApJ, 430, 839

\section{DISCUSSION}

Dziembowski: I am curious whether in your feeling the randomness we observe in the mode spectra rather reflects the stochastic nature of convection or rather mode interaction?

Montgomery: I would rather say mode interaction. The motions in the convection zone occur on such a small scale that they are well averaged out over the disk and over the modes that we observe, but I don't know for sure.

Mukadam: The hot PG 1159 stars exhibit nonlinear pulse shapes, similar to those shown by the $25000 \mathrm{~K}$ DB white dwarf pulsators. Kepler informed us yesterday that the convection zones in the hot PG 1159 stars are extremely thin. How can we understand the nonlinearities in the hot white dwarf pulsators?

Montgomery: Actually, while there may be significant beating between the excited modes in these objects, their pulse shapes are quite linear. This fits in nicely with the idea (from models) that these stars do not have surface convection zones.

Kiss: We know of many high-amplitude pulsating stars, like RV Tauris or Miras, which show strong nonlinear interactions and a relatively small number of modes. Do you see any restrictions which would prevent the use of your method in those cases?

Montgomery: The crucial assumption that makes this easier than other time-dependent convection formalisms, is that in our treatment the convection zone responds instantaneously to the flux perturbations. This is due to the fact that the convective turnover time is of order one second, while the pulsation modes have periods which are much longer, of order hundreds of seconds. I suspect that in the stars which you mention that this might not be the case. In other words, the mode period may be of the same order of magnitude as the 
convective turnover time and that's outside the range of this approximation. However, for some parameter ranges we might still be in the regime where we can learn something, so we might be able to go further than we think with this approximation.

Kepler: Did Brickhill or Goldreich \& Wu calculate a nonlinear energy contribution for the convectional driving of the pulsations?

Montgomery: They both did. This convective response is what drives things. I should mention to Wojtek and the other theorists that what I did is sort of an adiabatic version of what they did. I assumed that all the flux that goes into the convection zone eventually comes out of the top. It doesn't go into mechanical driving. In other words, the amplitudes have saturated. This is actually a slightly simpler version of their theory, but it gives support to what they did.

Bedding: You mentioned the differences between the models and the light curves. Wouldn't it be better to use your model to predict the combination frequencies and compare them to the combination frequencies of the observations?

Montgomery: I'm not sure that I would agree that this would be better, but yes, I think it is something we should also do.

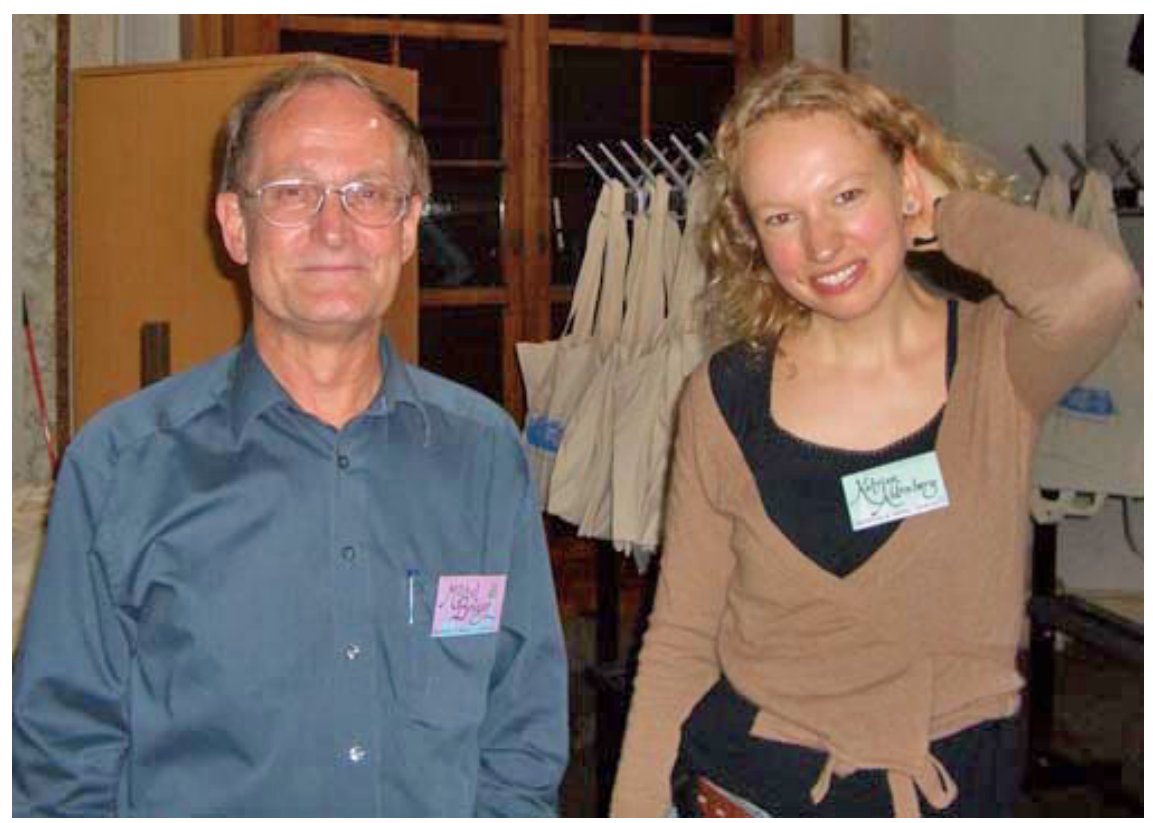

Michel Breger and Katrien Kolenberg. 